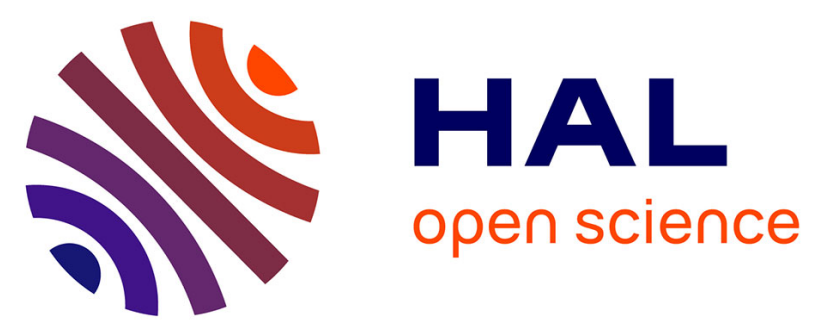

\title{
Fire retardancy effect of phosphorus-modified halloysite on polyamide-11 nanocomposites
}

Mohamed Sahnoune, Aurélie Taguet, Belkacem Otazaghine, Mustapha Kaci, José-Marie Lopez-Cuesta

\section{- To cite this version:}

Mohamed Sahnoune, Aurélie Taguet, Belkacem Otazaghine, Mustapha Kaci, José-Marie LopezCuesta. Fire retardancy effect of phosphorus-modified halloysite on polyamide-11 nanocomposites. Polymer Engineering and Science, 2019, 59 (3), pp.526-534. 10.1002/pen.24961 . hal-02424971

\section{HAL Id: hal-02424971 \\ https://hal.science/hal-02424971}

Submitted on 15 Dec 2020

HAL is a multi-disciplinary open access archive for the deposit and dissemination of scientific research documents, whether they are published or not. The documents may come from teaching and research institutions in France or abroad, or from public or private research centers.
L'archive ouverte pluridisciplinaire HAL, est destinée au dépôt et à la diffusion de documents scientifiques de niveau recherche, publiés ou non, émanant des établissements d'enseignement et de recherche français ou étrangers, des laboratoires publics ou privés. 


\title{
Fire Retardancy Effect of Phosphorus-Modified Halloysite on Polyamide-11 Nanocomposites
}

\author{
Mohamed Sahnoune, ${ }^{1,2}$ Aurélie Taguet, ${ }^{2}$ Belkacem Otazaghine, ${ }^{2}$ Mustapha Kaci, ${ }^{1}$ José-Marie Lopez-Cuesta ${ }^{2}$ \\ ${ }_{1}^{1}$ Laboratoire des Matériaux Polymères Avancés (LMPA), Université de Bejaia, 06000, Algeria \\ ${ }^{2}$ Centre des Matériaux des Mines d'Alès (C2MA), IMT Mines d'Alès, 6 avenue de Clavières, 30319, Alès cedex, France
}

\begin{abstract}
Halloysite nanotubes (HNTs) were successfully incorporated as flame retardants in polyamide-11 (PA11) after their modification with methyl phosphonic acid. Fourier transform infrared spectroscopy, thermal gravimetric analysis (TGA) and pyrolysis-gas chromatography-mass spectrometry were used to evidence the functionalization of the clay. Raw and modified HNTs were then incorporated by melt mixing in PA11 at 20 wt\%. Compositions containing both ammonium polyphosphate (APP) and HNTs were also prepared. TGA and pyrolysis combustion flow calorimeter exhibited enhancement in thermal stability upon incorporation of both raw and modified halloysite nanotubes while APP causes degradation at lower temperature. Cone calorimeter data showed that modified halloysite acts by forming an insulating barrier during the combustion, which limits heat and mass transfers. Moreover, elemental analysis of sample residues after cone test evidenced that a part of the phosphorus of the modified halloysite was transferred to the gaseous phase. These results suggest the full potential of halloysite as fire retardant agent for polyam-ides.
\end{abstract}

\section{INTRODUCTION}

Polyamide-11 (PA11) is a biocompatible commercial aliphatic polymer with excellent characteristics such as good oil resistance or low coefficient of friction and has the advantage to be less hydro-philic than the commonly used polyamide- 6 and $6,6[1,2]$. Therefore, PA11 is widely used as an engineering polymer in a large range of industrial fields from automotive to offshore applications including food packaging and sport equipments. Unfortunately, like all the polyamides, the fire behavior of PA11 is limited and applications in electric industries or in the building sector require the use of fire retardants. Several approaches to improve fire retardancy of polyamides can be envisaged, including the use of flame retardants additives $[3,4]$, grafting fire retardant groups on the polymer chain [5] or copolymerization with fire retardant monomers [6]. Nevertheless, most of current fire retardants cause destabilization of polyamides (discoloration and reduction in melt viscosity) because of sensitivity to acids, even at low concentrations [3]. Moreover, phenomena of migration of the additives during processing, water conditioning or ageing of the polymer are liable to entail loss of fire retardancy because of the undergone hydrolysis. Hence, nanocomposites technology has emerged as a route to improve the flammability of polyamides, not only with the use of nanoparticles alone, such as nanoclays [7-9], but more efficiently through synergies with flame retardants [10-13].

Correspondence to: A. Taguet; e-mail: aurelie.taguet@mines-ales.fr
Halloysite nanotubes (HNTs) are minerals of kaolinite group with the chemical composition $\mathrm{Al}_{2} \mathrm{Si}_{2} \mathrm{O}_{5}(\mathrm{OH})_{4} \cdot \mathrm{nH}_{2} \mathrm{O}$, which exhibit many advantages such as biocompatibility, thermal stability and reinforcing effect [14]. The literature reports numerous polymer/halloysite nanocomposite systems, leading to enhancements in mechanical properties, improvement of thermal stability, nucleating effect and also flame retardancy [15-18]. Indeed, it has been reported that halloysite, alone or in synergy with usual fire retardant, can enhance fire behavior of various polymers such as polypropylene [19-21], polyamide-6 [22], polylactic acid (PLA) [23], poly(butylene succinate) [24] or linear low-density polyethylene [25] through thermal insulation barriers that can be developed at the surface of the composite during the burning. It has been shown that this barrier reduces the mass loss rate, limits the heat release rate (HRR) and extends the time to flameout [22]. The encapsulation into the HNTs lumen of products resulting from the thermal degradation of the polymer is also known to contribute to the fire behavior enhancement of the nanocomposites [26]. Many factors have to be examined while considering nanoparticles as fire retardant, such as aspect ratio, shape or amount that can influence the barrier effect. To improve the flame retardant effect of halloysite, some authors have retained the strategy of chemical modification of the clay. For example, Li et al. [27] grafted maleic anhydride and 9,10-dihydro-9-oxa-10-phosphaphenanthrene-10-oxide (DOPO) at the surface of HNTs before adding it at $5 \mathrm{wt} \%$ in PLA. They reported that the improved fire retardancy was due to a slow release of DOPO derivations and a subsequent flame inhibition, together with secondary barrier effect. Marney et al. [28] intercalated halloysite with phenyl phosphonic acid, leading to an enhanced fire behavior of PA6/halloysite nanocomposites. Halloysite has unique tubular structure with aluminol groups at the inner surface and silanol ones at the outer. This difference of composition was used in this study to modify selectively inner surface of halloysite with methyl phosphonic acid (MPA). Indeed, the reactivity between HNTs aluminol groups and phosphonic functions allows the selective grafting of inner surface of HNTs [29,30]. In a recent article [31], organophosphorous flame retardant dimethyl methylphosphonate (DMMP) was loaded into HNTs lumen using vacuum (leading to HNT-D). A $20 \mathrm{wt} \%$ of HNT-D was added into UV-curable epoxy resin and a better flame retardancy was measured. The authors revealed that the flame retardant mechanism consisted in two steps: a first DMMP gasification followed by a polyphosphoric-HNTs carbon layer generation on the condensed phase. To the best of our knowledge, there are no other articles dealing with the effect of the chemical modification of HNTs on the flame retardancy of polymers, especially polyamides. 
Therefore, the main objective of this paper is to investigate the potential of halloysite, after its functionalization with MPA, as a fire retardant agent for PA11. Halloysite was used alone or in combination with a standard phosphorous fire retardant. The incorporation of the clay in PA11 was performed by melt compounding at $20 \mathrm{wt} \%$. Moreover, the results with the modified halloysite were compared to those of neat PA11 and PA11 containing raw halloysite and ammonium polyphosphate (APP).

\section{EXPERIMENTAL}

Materials

PA11 (Rilsan ${ }^{\circledR}$ LMNO) was kindly supplied by Arkema. This grade is free of any stabilizers or additives. Its mean molecular weight $\mathrm{Mn}=22,000 \mathrm{~g} / \mathrm{mol}$ and average molar weight $=51,000$ $\mathrm{g} / \mathrm{mol}$ with a density of $1.02 \mathrm{~g} / \mathrm{cm}^{3}$. Raw halloysite (referenced as Hal) derived from Djebel Debbagh deposit (Guelma, eastern region of Algeria). Halloysite particles having an average diameter of $25 \mu \mathrm{m}$ and a surface area of $51.4 \mathrm{~m}^{2} / \mathrm{g}$. This halloysite was characterized in details previously in [32]. MPA was purchased from ABCR. Less-hygroscopic APP Exolit 423 was kindly supplied from Clariant. Ethanol and acetone were purchased from Sigma Aldrich and Panreac, respectively. Before using, HNTs and polymer pellets were dried under vacuum at $80^{\circ} \mathrm{C}$ for $24 \mathrm{~h}$ and overnight, respectively, to remove moisture traces.

\section{Halloysite Functionalization}

The halloysite functionalization consisted of a reaction between halloysite and MPA. A $10 \mathrm{~g}$ of halloysite, $1 \mathrm{~g}$ of MPA and $100 \mathrm{ml}$ of an ethanol/water $(90 / 10 \mathrm{w} / \mathrm{w})$ solution were introduced into a $250 \mathrm{~mL}$ flask fitted with a condenser. The mixture was then stirred and heated at solvent reflux for $15 \mathrm{~h}$. The mixture was next centrifuged (speed: 5,000 rpm) to eliminate the liquid phase and washed three times with acetone. Finally, the obtained halloysite (referenced as Hal-P) was dried under vacuum.

\section{Samples Preparation}

Various compositions containing $20 \mathrm{wt} \%$ of Hal, Hal-P, APP, or a mixture of APP and Hal or Hal-P were prepared and Table 1 summarizes the studied compositions. The samples were prepared by melt mixing under vacuum in a semi-industrial twin screw extruder (BC 21 Clextral) at a screw speed of $250 \mathrm{rpm}$ with a screw diameter (D) of $25 \mathrm{~mm}$ and length to diameter ratio (L/D) of 48 . The temperature profile was $140^{\circ} \mathrm{C} / 250^{\circ} \mathrm{C} / 245^{\circ} \mathrm{C} / 235^{\circ} \mathrm{C} / 230^{\circ} \mathrm{C}$ from hopper to die. After pelletizing, the nanocomposites granules were dried under vacuum at $80^{\circ} \mathrm{C}$ overnight and then injection molded into standard test specimens for fire testing (plates of $100 \times 100 \times 4 \mathrm{~mm}^{3}$ ) using an injection molding machine KM50-180X (Krauss Maffei). The temperature profile setting ranged from $220^{\circ} \mathrm{C}$ to $225^{\circ} \mathrm{C}$ and the

TABLE 1. Code and composition of the samples

\begin{tabular}{ccccc}
\hline Samples & PA11 (wt $\%)$ & APP (wt $\%)$ & Hal (wt $\%)$ & Hal-P (wt $\%)$ \\
\hline PA11 & 100 & 0 & 0 & 0 \\
PA11/AP & 80 & 20 & 0 & 0 \\
PA11/H & 80 & 0 & 20 & 0 \\
PA11/HP & 80 & 0 & 0 & 20 \\
PA11/AP/H & 80 & 15 & 5 & 0 \\
PA11/AP/HP & 80 & 15 & 0 & 5 \\
\hline
\end{tabular}

mold temperature was kept at $40^{\circ} \mathrm{C}$. Holding pressure and screw rotation speed were 135 bar and $100 \mathrm{rpm}$, respectively, with a throughput of $50 \mathrm{~cm}^{3} / \mathrm{s}$.

\section{Characterization}

Fourier Transform Infrared Spectroscopy. The surface modification of halloysite after functionalization was investigated by Fourier transform infrared (FTIR) spectroscopy. FTIR was performed on a spectrometer IFS 66 (Bruker) on $\mathrm{KBr}$ pellets (1:99 ratio) and spectra were obtained by collecting 64 scans between 400 and $4,000 \mathrm{~cm}^{-1}$ with a resolution of $2 \mathrm{~cm}^{-1}$.

Pyrolysis-Gas Chromatography-Mass Spectrometry. A Pyroprobe 5000 pyrolyzer (CDS analytical) was used to flash pyrolyze the samples in a helium environment. This pyrolyzer is equipped with an electrically heated platinum filament. One coil probe enables the pyrolysis of samples $(<1 \mathrm{mg})$ placed in a quartz tube between two pieces of rockwool. The sample was heated at $900^{\circ} \mathrm{C}$. The temperature was held for $15 \mathrm{~s}$, and then the gases were drawn to the gas chromatograph for $5 \mathrm{~min}$. The pyroprobe 5000 is interfaced to a $450-\mathrm{GC}$ chromatograph (Varian) by means of a chamber heated at $270^{\circ} \mathrm{C}$. In the oven, the initial temperature of $70^{\circ} \mathrm{C}$ was raised to $250^{\circ} \mathrm{C}$ at $10^{\circ} \mathrm{C} / \mathrm{min}$. The column is a Varian Vf-5 ms capillary column $(30 \mathrm{~m} \times 0.25 \mathrm{~mm}$; thickness $=0.25$ $\mu \mathrm{m})$ and helium $(1 \mathrm{~L} / \mathrm{min})$ was used as the carrier gas, a split ratio was set to 1:50. The gases were introduced from the GC transfer line to the ion trap analyzer of the 240-MS mass spectrometer (Varian) through the direct-coupled capillary column.

X-Ray Diffraction. The crystalline structure of the specimens was examined using wide angle XRD measurements. An X-ray diffractometer (XRD) Bruker D8 (Bruker, USA) equipped with a $\mathrm{Cu}-\mathrm{K} \alpha$ radiation source $\left(\lambda=1.5418 \mathrm{~A}^{\circ}\right)$ and operated at $30 \mathrm{kV}$ and $40 \mathrm{~mA}$, was used for the investigations in the interval range $2 \vartheta$ from $5^{\circ}$ to $70^{\circ}$ with a scanning step of $0.007^{\circ}$.

Thermogravimetric Analysis. The thermal stability of the halloysites and the nanocomposite samples was studied by thermal gravimetric analysis (TGA). The analyses were performed by a thermogravimetric analyzer (Setsys Evolution, Setaram). In order to remove all the physisorbed water of the halloysites, an isothermal step $\left(10 \mathrm{~min}, 110^{\circ} \mathrm{C}\right)$ was performed before starting the analysis and then the samples were heated to $900^{\circ} \mathrm{C}$ at a heating rate of $10^{\circ} \mathrm{C} / \mathrm{min}$, while the nanocomposite samples were heated to $600^{\circ} \mathrm{C}$ at $10^{\circ} \mathrm{C} / \mathrm{min}$. Measurements were carried out under air or nitrogen atmospheres with a flow rate of $20 \mathrm{~mL} / \mathrm{min}$ on samples of $\sim 20 \mathrm{mg}$.

Transmission Electron Microscopy. Transmission electron microscopy (TEM) was conducted to evaluate the degree of dispersion of both raw and modified HNTs in the PA11 matrix. Micrographs were obtained with a JEOL 1200EX TEM (JEOL) operating at an accelerating voltage of $100 \mathrm{kV}$. The TEM samples were embedded in a LR white resin and ultrathin-sectioned at $70 \mathrm{~nm}$ using a Leica EM UC7 ultra-microtome with a diatome diamond knife Ultra 45 (Nissei Sangyo). Sections were collected on 300 mesh copper grids coated with carbon film. 
Scanning Electron Microscopy. Scanning electron microscopy (SEM) was conducted under high vacuum with an environmental SEM Quanta 200 FEG (FEI Company) operating at $12.5 \mathrm{kV}$. It was first used to investigate the morphology of the PA11/HNTs samples. Injection molded test specimens were cryo-fractured and coated with a thin carbon layer. The fracture surfaces were observed in the back-scattered electrons mode. Moreover, SEM was used to observe the residues collected after cone calorimeter test for PA11/H and PA11/HP.

Pyrolysis Combustion Flow Calorimeter. Pyrolysis combustion flow calorimeter (PCFC) has been used to study the fire behavior of mg-sized samples. PCFC analysis was carried out according to the method A (anaerobic pyrolysis) of the ASTM D7309 using a Fire Testing Technology (FTT) apparatus with a heating rate of $1^{\circ} \mathrm{C} / \mathrm{s}$, the maximum pyrolysis temperature was $750^{\circ} \mathrm{C}$ and the combustion temperature was $900^{\circ} \mathrm{C}$ (corresponding to complete combustion). The flow in combustor was a mixture of $\mathrm{O}_{2} / \mathrm{N}_{2} 20 / 80$ at $100 \mathrm{~cm}^{3} \cdot \mathrm{min}^{-1}$ and the sample weight was $0.5-2 \mathrm{mg}$.

Cone Calorimeter. The fire behavior of the studied compositions was mainly studied using a FTT cone calorimeter. The measurements were carried out according to ISO 5660-1 test procedure at incident heat flux of $50 \mathrm{~kW} / \mathrm{m}^{2}$ and the ignition of the flame was initiated using a spark igniter. The cone data reported here are averages of at least three replicates reproducible with variation $<10 \%$. The fire property parameters considered were HRR, peak of HRR (PHRR), total heat released (THR), maximum average rate of heat emission (MAHRE), and time to ignition (TTI).

Elemental Analysis. The phosphorus content was measured by inductively coupled plasma atomic emission spectroscopy (ICP-AES). Measurements were carried out by SGS Multilab. Modified halloysite (Hal-P) and the residue after cone calorimeter test of PA11/HP were analyzed. Hal-P contains $16 \mathrm{wt} \%$ of water whereas the residue of PA11/HP is in the dehydrated form. Hence, water was removed in the calculation of the final phosphorus content of Hal-P and the dry mass of phosphorus (wt\% of $\mathrm{P}$ ) is given in the Results and Discussion part.

\section{RESULTS AND DISCUSSION}

Characterization of the Functionalization

The purpose of the functionalization is to confer a better flame retardancy action through the modification of halloysite using a phosphorous compound, the MPA. The functionalization is assumed to be performed on the inner surface of HNTs, since phosphonic compounds can only react with aluminol groups present at the inner surface [29]. Modified HNTs (Hal-P) were obtained after several steps of centrifugation and washing in order to eliminate all the MPA molecules that were not covalently attached to the aluminol groups of the HNTs. Figure 1 shows FTIR spectra of Hal, Hal-P and MPA. A weak band can be observed at $1,320 \mathrm{~cm}^{-1}$ for Hal-P (Fig. 1b). This additional band is attributed to the alkyl group of the MPA [33]. It is difficult to observe new bands, even in the transmission mode, because of the localization of the MPA at the inner surface. Indeed, the peak absorbance shifts toward lower values due to the more constricted
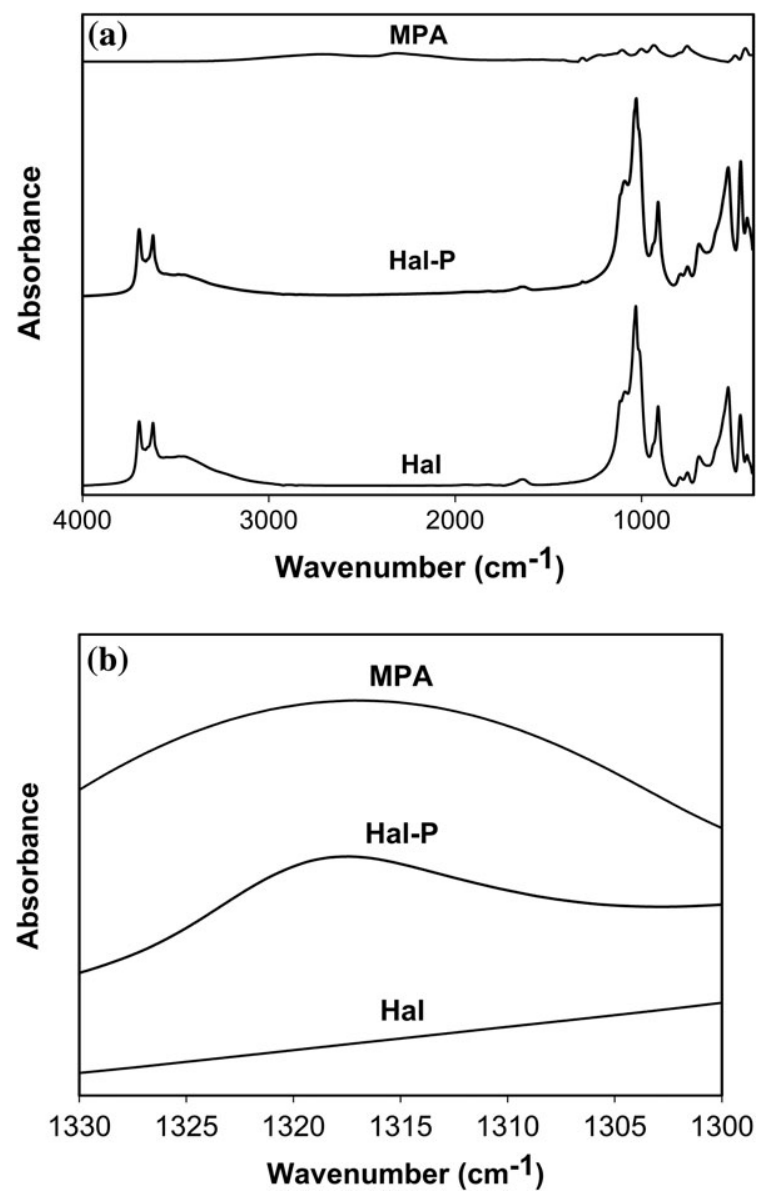

FIG. 1. FTIR spectra of Hal, Hal-P and MPA (a). The region between 1,300 and $1,330 \mathrm{~cm}^{-1}$ (b).

vibration of alkyl groups inside the confined spaces of halloysite lumen [29,30].

Pyrolysis-gas chromatography-mass spectrometry (Py-GC/ MS) was also used to evidence the functionalization and the obtained results are presented in Fig. 2. The first observation after halloysite modification concerns the main observed peak at $1.5 \mathrm{~min}$ in Hal that is assigned to a sulfonate fraction resulting from a pollution due to the raw nature of the used HNTs. We

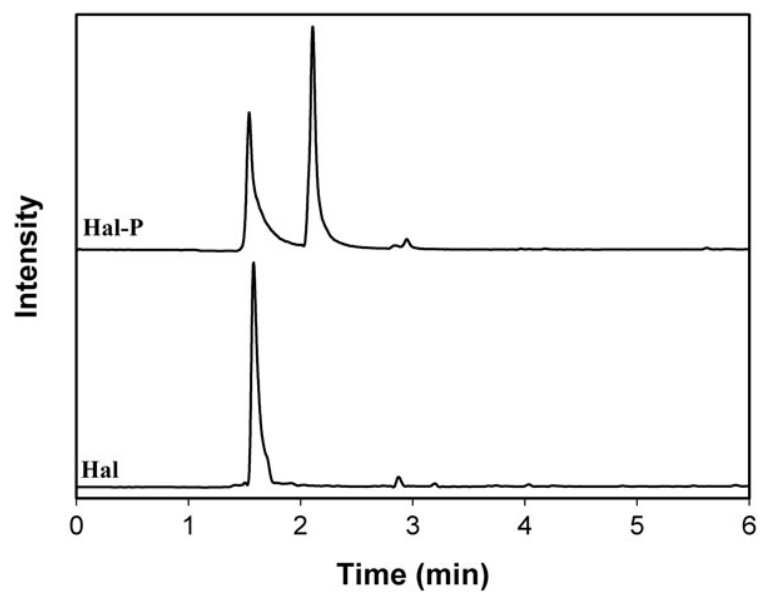

FIG. 2. Py-GC/MS chromatograms of Hal and Hal-P. 


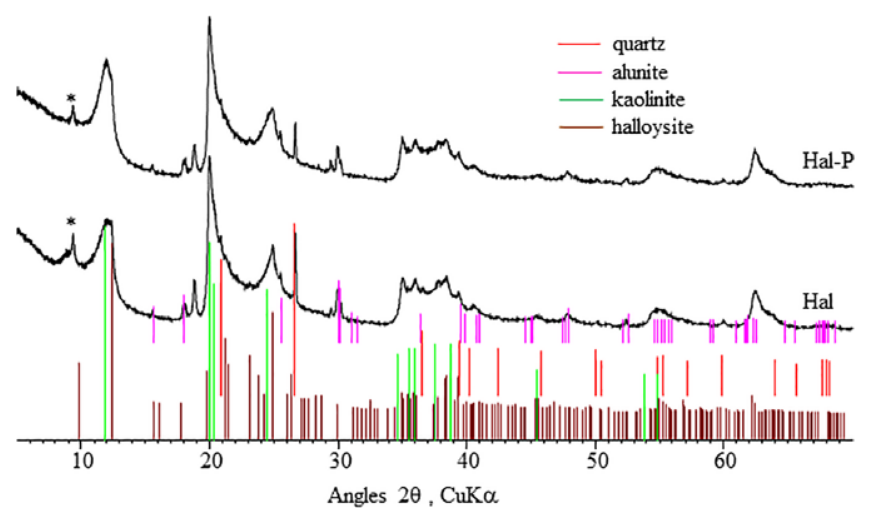

FIG. 3. XRD patterns of Hal and Hal-P. [Color figure can be viewed at wileyonlinelibrary.com]

observe the appearance of a new peak at 2.1 min corresponding to another sulfur species, which can be the result of a combination between the decomposition products of MPA and the sulfur fraction of raw halloysite during the pyrolysis step.

The XRD patterns of the samples (Fig. 3) confirm the presence of halloysite (basal reflection at $2 \vartheta=12^{\circ}$ ) in its tubular form (very intense reflection at about $20^{\circ}$ ) with presence of some other mineral impurities like quartz and calcite [34]. As both XRD spectra of Hal and Hal-P matches, it is shown that MPA does not change the crystalline structure of the nanotubes, as the peaks remain unchanged even after functionalization. This proves that MPA is not intercalated but either encapsulated or grafted onto the lumen surface or grafted at the edge of the nanotubes [28,35].

TGA curves of the clay samples are displayed in Fig. 4. Experiments were conducted under air because halloysite modified with a phosphonic acid molecule has a tendency to react with nitrogen. As evidenced, both halloysites show a first step of thermal decomposition up to $230{ }^{\circ} \mathrm{C}$ due to the release of the remaining physisorbed water (surface and interlayer) [36]. The main mass loss corresponding to the structural dehydroxylation of the hydroxyl groups of the surface of HNTs occurs between $400^{\circ} \mathrm{C}$ and $570^{\circ} \mathrm{C}$ [36]. Hal-P features a slightly more pronounced loss, which was attributed to the decomposition of the grafted organic part. This slight difference is due to the non-complete degradation of the MPA molecules that have lost only around $37 \%$ of their weight at $900^{\circ} \mathrm{C}$, under air.

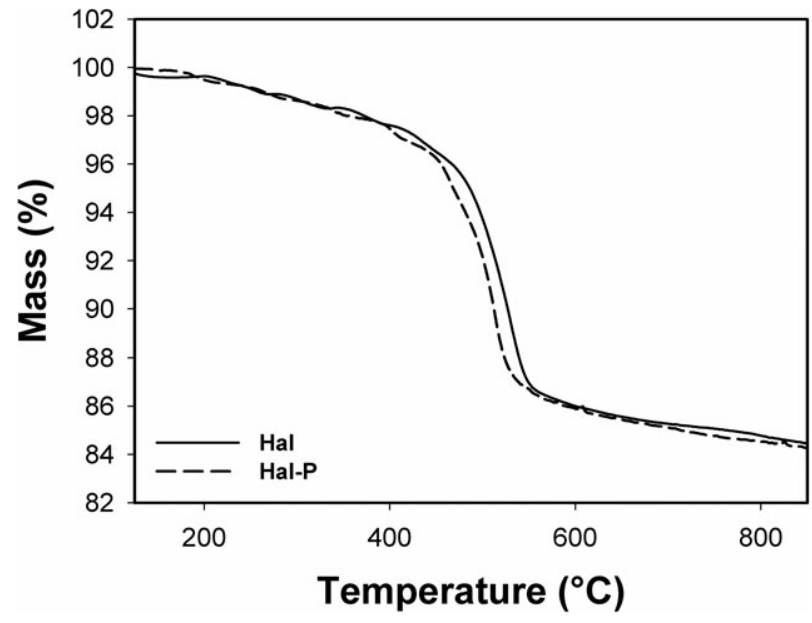

FIG. 4. TGA curves of Hal and Hal-P under air.
Hence, this additional mass loss for Hal-P compared with Hal does not allow calculating accurately a grafting rate of MPA.

\section{Morphology}

The dispersion of both raw and modified HNTs was first evaluated by TEM. The pictures (Fig. 5) reveal the individual dispersion of the two HNTs at the nanoscale in the PA11 matrix. Furthermore, SEM micrographs of the fractured surface of PA11/H and PA11/HP nanocomposites are presented in Fig. 6. The images confirm the homogeneous and uniform dispersion of both Hal and Hal-P in the PA11 matrix. However, some small bundles of HNTs are also visible but they are less than $15 \mu \mathrm{m}$ in size. They correspond in fact to the micronic particles resulting from the comminution process of halloysite, which does not allow a complete release of nanotubes to be performed, especially at high filler content that is $20 \mathrm{wt} \%$.

The morphological investigations through TEM and SEM indicate clearly that halloysite modification through MPA did not influence the state of dispersion of the clay in the matrix when incorporated at $20 \mathrm{wt} \%$.

\section{Thermal Stability of the Nanocomposites}

The thermal and fire behavior of PA11 containing $20 \mathrm{wt} \%$ of Hal and Hal-P was compared with those containing $20 \mathrm{wt} \%$ of APP and a mixture between APP and Hal or Hal-P. The thermal stability of the different compositions was investigated mainly by TGA. TGA and differential thermogravimetric (DTG) curves obtained under nitrogen and air atmospheres are shown in Figs. 7 and 8 , respectively.

At first, in non-oxidant atmosphere a decrease in PA11 thermal stability in presence of APP is noticed. Thus, the temperature of the maximum rate of degradation, corresponding to the peak of the DTG curves, is reduced by $60^{\circ} \mathrm{C}$ compared with neat PA11, whereas halloysite improves thermal stability by $15^{\circ} \mathrm{C}$. Intermediate results are observed when APP is partially substituted by HNTs but with a better effect in the presence of modified halloysite.

Under oxidative atmosphere, the same trends are observed as APP causes earlier degradation $\left(-72^{\circ} \mathrm{C}\right)$ while halloysite enhances thermal stability of PA11 matrix. Nevertheless, the improvement of thermal stability is only significant in presence of modified halloysite $\left(+14^{\circ} \mathrm{C}\right.$ for the temperature of maximum degradation rate).

The early degradation of the systems containing APP (whatever the atmosphere) is due to release of water from APP and to the formation of phosphoric acid that causes PA11 hot hydrolysis and advanced thermal decomposition [37]. Conversely, it is suggested that the enhancement of the thermal stability in presence of halloysite is due to its high specific surface area, which restricts the motion of macromolecular species when the polymer decomposes. Moreover, its tubular structure with large lumen volume may entrap some degradation products, resulting in an effective delay in mass transfer of volatile decomposition products [19].

PCFC measurements under inert and oxidative atmospheres are shown in Fig. 9a and b, respectively.

Under anerobic atmosphere (nitrogen), a significant influence on degradation in presence of APP is observed, as the temperature of the pHRR decreases by $40{ }^{\circ} \mathrm{C}$ in comparison to neat PA11. In addition, a higher value of $\mathrm{pHRR}$ is noticed $(667 \mathrm{~W} / \mathrm{g}$ for neat PA11 to $1060 \mathrm{~W} / \mathrm{g}$ for PA/AP), whereas, incorporation of raw or 

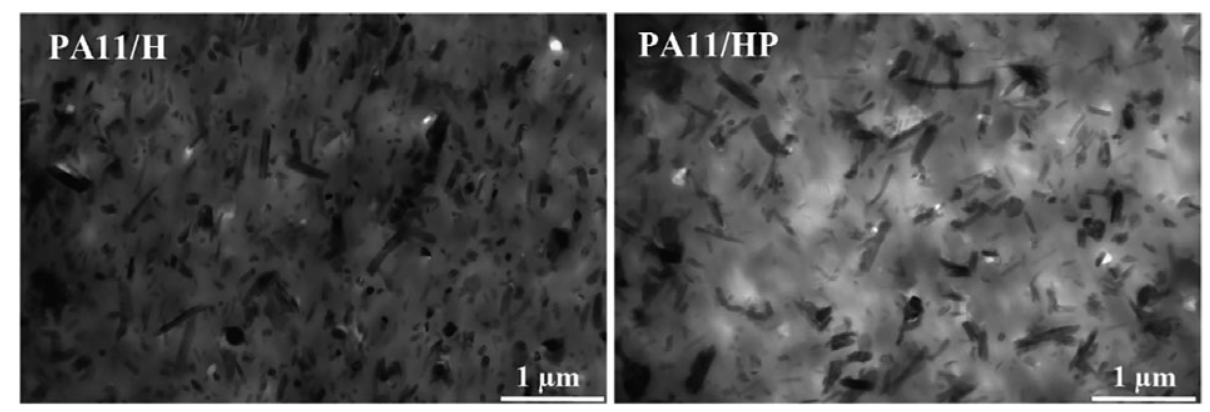

FIG. 5. TEM pictures of PA11/H and PA11/HP.

modified halloysite, delays PA11 degradation $\left(+33^{\circ} \mathrm{C}\right)$ but with slightly higher values of pHRR. Under oxygen, a degradation at lower temperature is also noticed when APP is present in the compositions. Halloysite incorporation leads to better thermal stability but only Hal-P induces a significant reduction of pHRR from $1255 \mathrm{~W} / \mathrm{g}$ for virgin PA11 to $676 \mathrm{~W} / \mathrm{g}$ for PA11/HP.

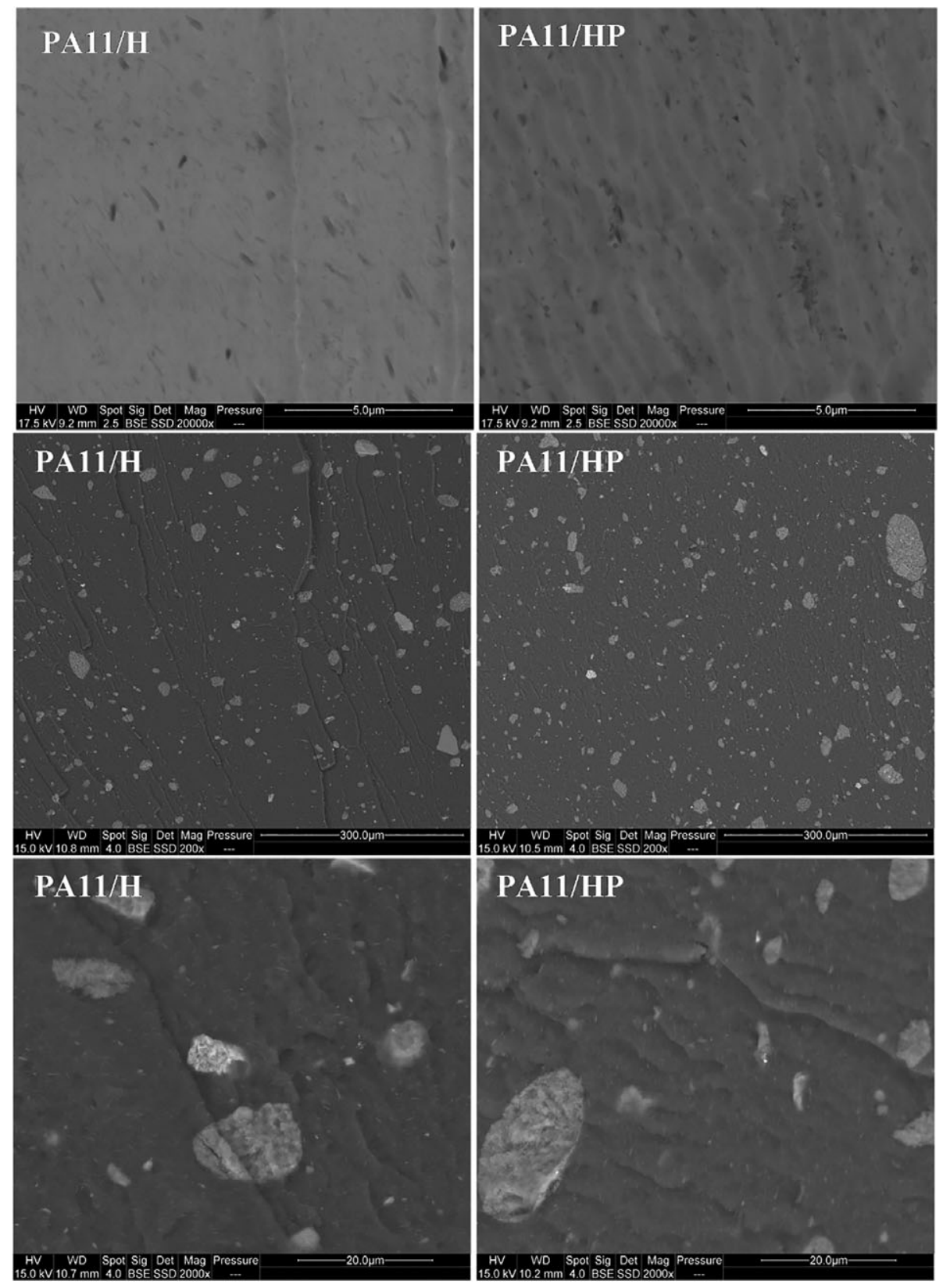

FIG. 6. SEM micrographs of fractured surfaces of PA11/H and PA11/HP under different magnifications. 

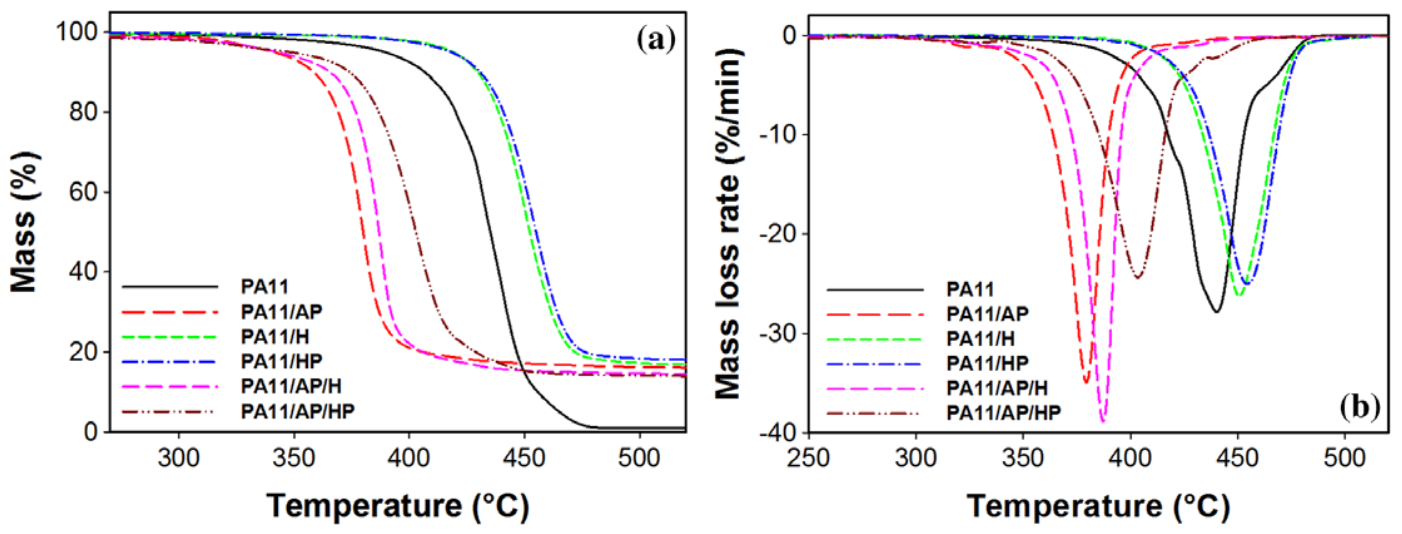

FIG. 7. TGA (a) and DTG (b) curves of the different compositions obtained at $10{ }^{\circ} \mathrm{C} / \mathrm{min}$ under nitrogen. [Color figure can be viewed at wileyonlinelibrary.com]

As the pHRR of PA11/HP is almost the same whatever the atmosphere, it means that Hal-P may limit oxygen diffusion through the nanocomposite during thermal degradation. Moreover, a reduction in pHRR when $5 \mathrm{wt} \%$ of APP is substituted by Hal-P is also observed, in comparison with APP alone. Since it has been shown that PCFC test does not take into account physical effects [38,39], we can argue that the decrease in pHRR with incorporation of Hal-P can be also due to the substitution of a fraction of the organic polymer matrix by halloysite or catalytic effects able to modify the degradation pathway of the polymer [40].

\section{Cone Calorimeter}

Fire behavior of the different compositions was mainly studied by cone calorimeter and the heat release curves at $50 \mathrm{~kW} / \mathrm{m}^{2}$ heat flux are illustrated in Fig. 10. The cone calorimeter collected data are listed in Table 2.

From Fig. 10, it can be seen that most of the HRR curves are very sharp, indicating intense burning and strong heat release, except for PA11/HP, when the incorporation of $20 \mathrm{wt} \%$ of modified halloysite to the matrix leads to a flat HRR curve with a time to flame out much longer than that of neat PA11. This plateau of HRR, corresponding to a thick charring behavior [41], is ascribed to the formation of a strong protective char layer that lasts longer before breaking during the combustion. The presence of $20 \mathrm{wt} \%$ of APP enhances pHRR by $9 \%$ while all the other compositions lead to a decrease in pHRR in comparison to the neat PA11 with the better results for PA11/HP $(-57 \%)$.

The cone calorimetric data (Table 2) confirm that the flammability of PA11/HP was decreased in comparison to the other compositions, as both the time of flameout (TOF) and the MAHRE of the formulation are all optimized compared to those of neat PA11. The addition of any flame retardant systems (APP, HNTs or both) decreases the TTI in Table 2 because the release of water and phosphoric acid (in the case of APP) involves hydrolysis of the PA11. The observation of a slightly lower char yield than the original mass of HNTs incorporated into the composites is anticipated, as HNTs are expected to lose their associated water, and undergo de-hydroxylation [42]. However, a higher amount of residue is found for PA11/HP in comparison to PA11/H, which can be ascribed to an incomplete pyrolysis due to char promotion through catalytic effects in presence of Hal-P (images of the residue are available in Supporting Information).

From the results, it can be considered that APP does not improve fire retardancy of PA11. This is probably due, in our case to the amount of APP that was not optimized to reach the best flame retardant effect for PA11. Moreover, the stability of the protective char layer formed by APP is limited because of both PA11 hydrolysis due to water release and the formation of phosphoric acid during APP degradation [11]. Indeed, it is known that APP acts in the condensed phase via a protective char during combustion [43]. In the case of Hal-P, a cohesive protective char layer is
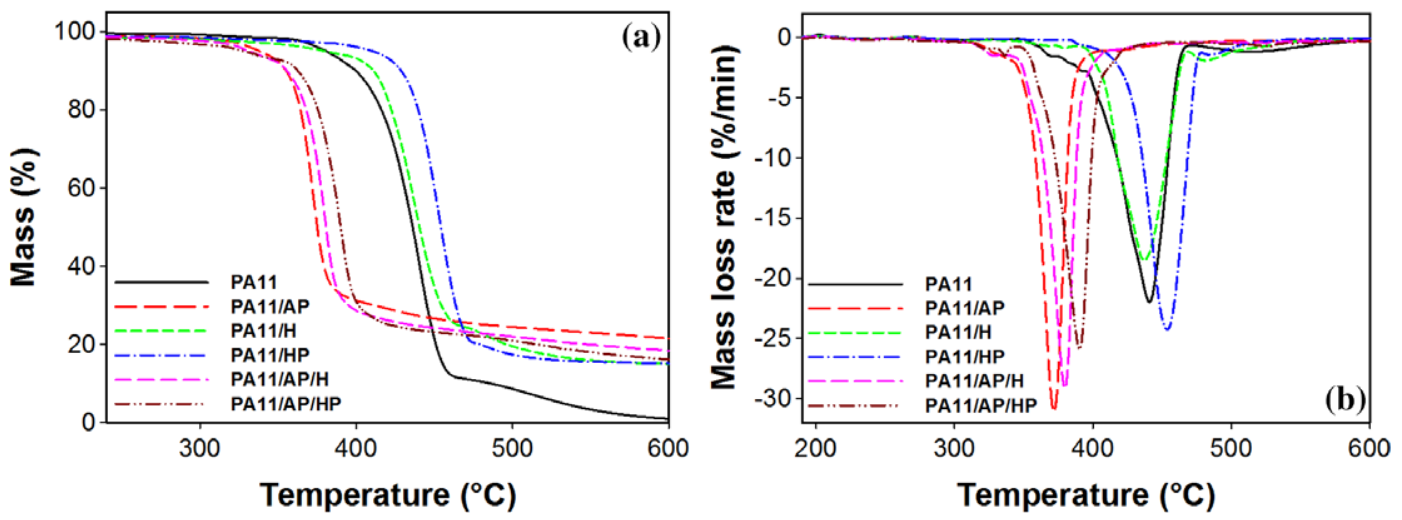

FIG. 8. TGA (a) and DTG (b) curves of the different compositions obtained at $10{ }^{\circ} \mathrm{C} / \mathrm{min}$ under air. [Color figure can be viewed at wileyonlinelibrary.com] 

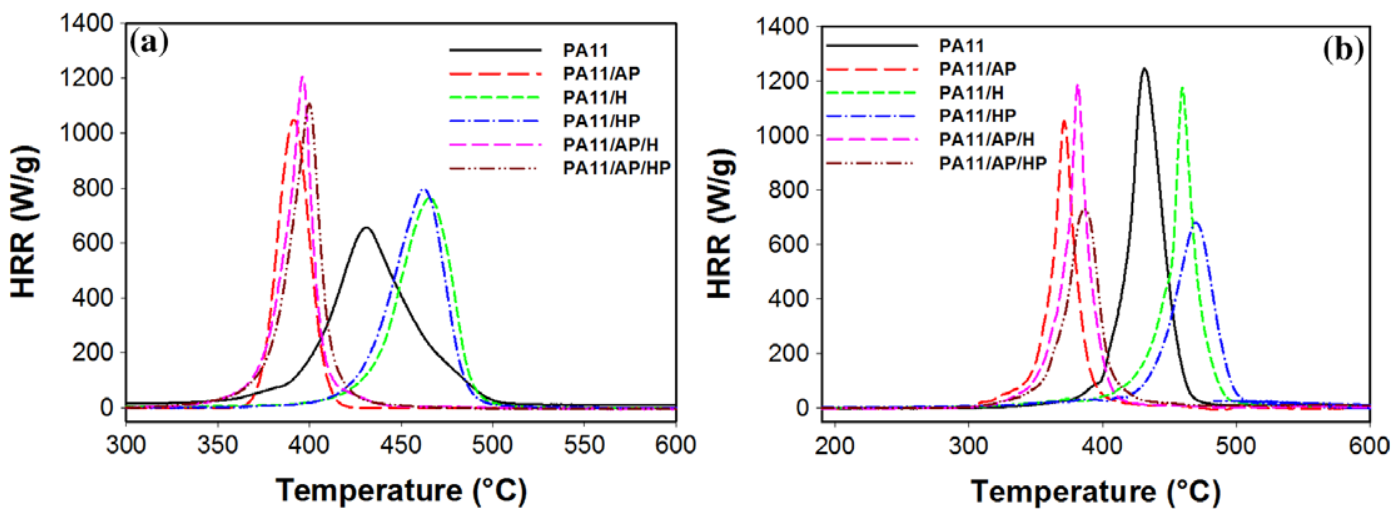

FIG. 9. PCFC curves under nitrogen (a) and air (b) of the different compositions. [Color figure can be viewed at wileyonlinelibrary.com]

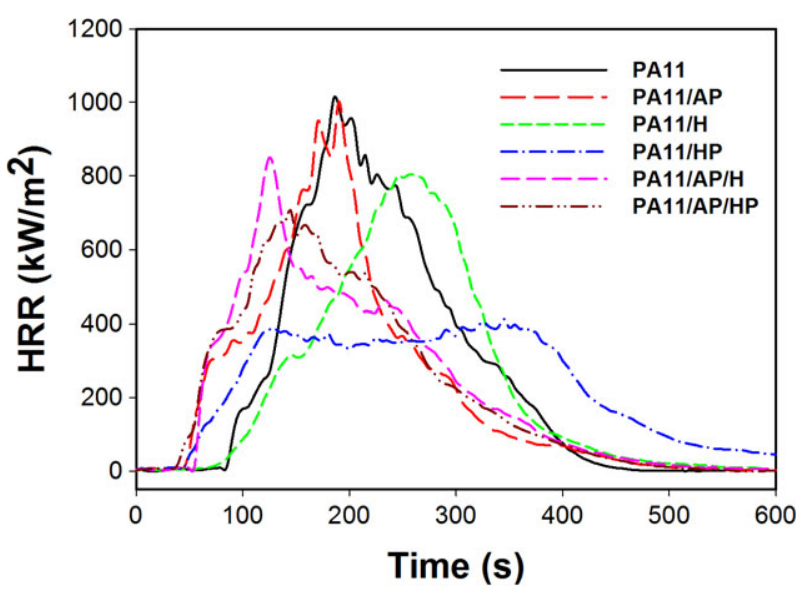

FIG. 10. HRR as function of time for the different compositions at a heat flux of $50 \mathrm{~kW} / \mathrm{m}^{2}$. [Color figure can be viewed at wileyonlinelibrary.com]

formed during the combustion, which does not break down and forms an insulating layer between the melted polymer and the flame zone. This acts to reduce the heat and mass transfer between the gaseous (flame zone) and condensed phase (melted polymer) [22], thereby slowing down the pyrolysis process and consequently lowering the heat release rate (pHRR).

The substitution of $5 \mathrm{wt} \%$ of APP by HNTs, raw or modified, results in an improvement of the fire reaction in comparison to neat PA11 and PA11/AP due to an additive effect of halloysite. It is particularly evident for PA11/AP/HP (containing $5 \mathrm{wt} \%$ of Hal-P) in comparison with PA11/AP/H and PA11/AP. Hence, a reinforcing effect of the charred structure promoted by APP owing to the presence of HNTs could be considered. Moreover, reactions between halloysite and APP degradation products to
TABLE 3. wt $\%$ of $\mathrm{P}$ obtained by elemental analysis (ICP-AES)

\begin{tabular}{ccc}
\hline Samples & Results from ICP-AES (mg/kg) & wt\% of P \\
\hline Hal-P & 20587 & 2.06 \\
Residue of PA11/HP & 18621 & 1.86 \\
\hline
\end{tabular}

form aluminophosphates that will stabilize the char layer at high temperature can be assumed [24,44].

When analyzing the results it appears that the MPA functionalization exerts a critical influence on the fire behavior. This is consistent with the literature where it was found that phenyl phosphonic acid modified halloysite can disrupt both the pyrolysis and combustion process in PA6/halloysite nanocomposites [28].

\section{Residue Analysis}

Some analyses were undertaken on the cone calorimeter residues. First, elemental analysis was carried out to quantify both the amount of phosphorus present on Hal-P and remaining in the residue of the samples containing the modified halloysite. The results (Table 3) indicate that Hal-P contains 2.06 wt\% of phosphorus, whereas the residue after cone calorimeter test of PA11/HP contains $1.86 \mathrm{wt} \%$. This difference can be due to the transfer of some phosphorus content in the gaseous phase during the combustion, which can suppose an effect of Hal-P in the gaseous phase.

The residues for PA11/H and PA11/HP were also collected at the middle-time of the cone calorimeter test with degraded and non-degraded parts. The SEM micrographs of these samples noted PA11/H-middle and PA11/HP-middle, respectively are given in Fig. 11. For PA11/H-middle, a clearly straight limit between the burned material (at the top) and the unspoiled

TABLE 2. Cone calorimeter data at heat flux of $50 \mathrm{~kW} / \mathrm{m}^{2}$

\begin{tabular}{cllcccc}
\hline & TTI $(\mathrm{s})$ & TOF $(\mathrm{s})$ & pHHR $\left(\mathrm{kW} / \mathrm{m}^{2}\right)$ & Time pHRR $(\mathrm{s})$ & MAHRE $\left(\mathrm{kW} / \mathrm{m}^{2}\right)$ & THR $\left(\mathrm{MJ} / \mathrm{m}^{2}\right)$ \\
\hline PA11 & $88 \pm 2$ & $440 \pm 29$ & $977 \pm 30$ & $190 \pm 5$ & $440 \pm 10$ & $152 \pm 2$ \\
PA11/AP & $52 \pm 3$ & $543 \pm 10$ & $1067 \pm 65$ & $195 \pm 5$ & $452 \pm 21$ & $138 \pm 2$ \\
PA11/H & $65 \pm 7$ & $543 \pm 57$ & $816 \pm 90$ & $255 \pm 10$ & $365 \pm 33$ & $139 \pm 3$ \\
PA11/HP & $53 \pm 5$ & $680 \pm 2$ & $416 \pm 3$ & $255 \pm 90$ & $288 \pm 5$ & 15.9 \\
PA11/AP/H & $44 \pm 12$ & $609 \pm 48$ & $825 \pm 23$ & $130 \pm 5$ & $411 \pm 21$ & 13.2 \\
PA11/AP/HP & $48 \pm 7$ & $550 \pm 21$ & $726 \pm 32$ & $152 \pm 8$ & $404 \pm 4$ & $135 \pm 1$ \\
\hline
\end{tabular}



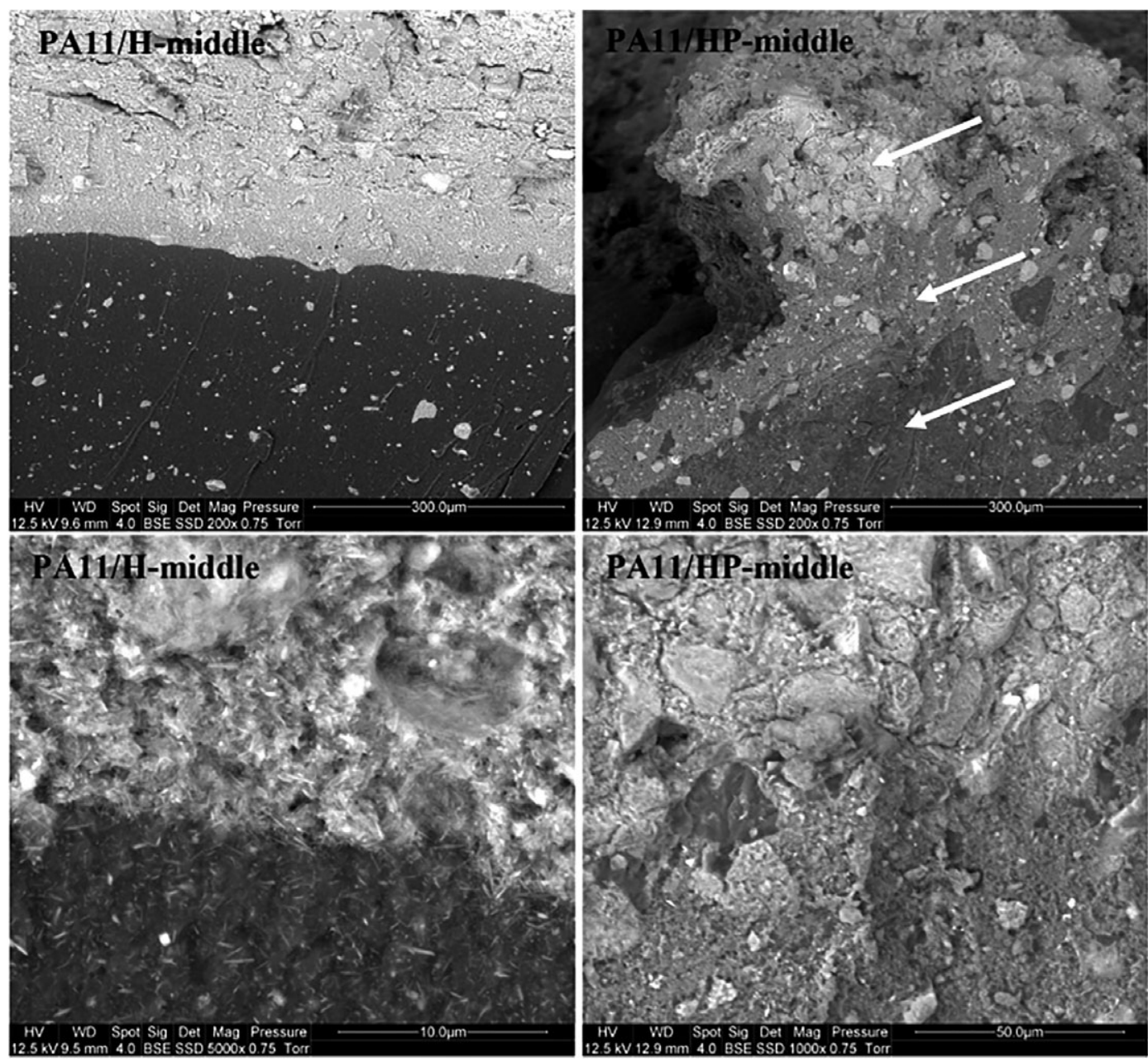

FIG. 11. SEM micrographs of the residues collected at the middle-time of the cone calorimeter test for PA11/H and PA11/HP. Low magnification (up) and high magnification (down).

one is visible, meaning that the pyrolysis front advances straight. For the PA11/HP-middle sample, the microstructure is highly different as different zones can be noticed (at least three zones pointed by three arrows). We assume that the phosphorous compound grafted on Hal-P reacts with the PA11 beside, to form a more cohesive residue that impedes the pyrolysis to progress straight ahead. As seen by the microstructure of the upper burned part of the PA11/HP-middle sample, it is considered that phosphorus also reacts with aluminum leading to an aluminum phosphate $\left(\mathrm{AlPO}_{3}\right)$ [45].

\section{CONCLUSIONS}

Halloysite modification with methyl phosphonic acid was successfully performed and FTIR, TGA, and Py-GC/MS analyses evidenced that functionalization. The influence of the halloysite modification on thermal stability and fire behavior of PA11 nanocomposites was then investigated in comparison to those of composites filled with raw halloysite, APP and mixed compositions. Halloysite, especially the modified one, improves thermal stability of the PA11 in both inert and oxidative atmospheres while APP induces thermal degradation. Moreover, compared with APP that releases phosphoric acid at temperatures $<300^{\circ} \mathrm{C}$, the phosphonic acid grafted on the HNTs allows PA11 flammability to be improved by the formation of an insulating and cohesive char barrier. This strong barrier due to the formation of aluminum phosphate does not break during the combustion and prevents heat and mass transfer between gaseous and condensed phases. These results suggest the full potential of halloysite after its functionalization to become an effective and environment-friendly fire retardant agent.

\section{ACKNOWLEDGMENTS}

The authors are grateful to EGIDE for its financial support through the Tassili program 13MDU891, to Jean-Jacques Flat from Arkema, and Soalka for supplying PA11 and raw Algerian halloysite, respectively. The authors are also thankful to Benjamin Gallard and Loic Dumazert of C2MA for their help during samples elaboration and characterization, respectively.

\section{REFERENCES}

1. X. He, J. Yang, L. Zhu, B. Wang, G. Sun, P. Lv, I.Y. Phang, and T. Liu, J. Appl. Polym. Sci., 102, 549 (2006).

2. Y. Liu, Z. Feng, and Q. Wang, Polym. Compos., 30, 221 (2009).

3. S.V. Levchik and E.D. Weil, Polym. Int., 49, 1033 (2000).

4. K.S. Lim, S.T. Bee, L.T. Sin, T.T. Tee, C.T. Ratnam, D. Hui, and A.R. Rahmat, Compos. Part B Eng., 84, 155 (2016).

5. V. Girardon, M. Tessier, and E. Maréchal, Polym. Bull., 33, 657 (1994). 
6. S.W. Shalaby, S. Sifniades, and D. Sheehan, J. Polym. Sci. Polym. Chem. Ed., 14, 2675 (1976).

7. P. Kiliaris and C.D. Papaspyrides, Prog. Polym. Sci., 35, 902 (2010).

8. M. Batistella, A.S. Caro-Bretelle, B. Otazaghine, P. Ienny, R. Sonnier, C. Petter, and J.M. Lopez-Cuesta, Appl. Clay Sci., 8, 116 (2015).

9. A.D. Macheca, W.W. Focke, M. Kaci, B. Panampilly, and R. Androsch, Polym. Eng. Sci. (2017). https://doi.org/10.1002/pen. 24775

10. L. Song, Y. Hu, Z. Lin, S. Xuan, S. Wang, Z. Chen, and W. Fan, Polym. Degrad. Stab., 86, 535 (2004).

11. J.B. Dahiya, S. Rathi, H. Bockhorn, M. Hauffmann, and B.K. Kandola, Polym. Degrad. Stab., 97, 1458 (2012).

12. A. Hao, I. Wong, H. Wu, B. Lisco, B. Ong, A. Sallean, S. Butler, M. Londa, and J.H. Koo, J. Mater. Sci., 50, 157 (2015).

13. B. Schartel and G. Schmaucks, Polym. Eng. Sci., 57, 1099 (2017).

14. M. Du, B. Guo, and D. Jia, Polym. Int., 59, 574 (2010).

15. U.A. Handge, K. Hedicke-Höchstötter, and V. Altstädt, Polymer., 51, 2690 (2010).

16. K. Prashantha, H. Schmitt, M.F. Lacrampe, and P. Krawczak, Compos. Sci. Technol., 71, 1859 (2011).

17. B. Lecouvet, M. Sclavons, S. Bourbigot, and C. Bailly, Polym. Adv. Technol., 25, 137 (2014).

18. J. Sun, X. Gu, M. Coquelle, S. Bourbigot, S. Duquesne, M. Casetta, and S. Zhang, Polym. Adv. Technol., 25, 1552 (2014).

19. M. Du, B. Guo, and D. Jia, Eur. Polym. J., 42, 1362 (2006).

20. A. Subasinghe, R. Das, and D. Bhattacharyya, Int. J. Smart Nano Mater., 7, 202 (2016).

21. W. Sun, W. Tang, X. Gu, S. Zhang, J. Sun, H. Li, and X. Liu, J. Appl. Polym. Sci., 135, 1 (2018).

22. D.C.O. Marney, L.J. Russell, D.Y. Wu, T. Nguyen, D. Cramm, N. Rigopoulos, N. Wright, and M. Greaves, Polym. Degrad. Stab., 93, 1971 (2008).

23. G. Stoclet, M. Sclavons, B. Lecouvet, J. Devaux, P. Van Velthem, A. Boborodea, S. Bourbigot, and N. Sallem-Idrissi, RSC Adv., 4, 57553 (2014).

24. Y. Wang, C. Liu, X. Shi, J. Liang, Z. Jia, and G. Shi, Polym. Compos. (2017). https://doi.org/10.1002/pc.24629.

25. Z. Jia, Y. Luo, B. Guo, B. Yang, M. Du, and D. Jia, Polym. Plast. Technol. Eng., 48, 607 (2009).
26. K. Szpilska, K. Czaja, and S. Kudla, Polimery., 12, 673, 673 (2015).

27. Z. Li, D. Fernández Expósito, A. Jiménez González, and D.Y. Wang, Eur. Polym. J., 93, 458 (2017).

28. D.C.O. Marney, W. Yang, L.J. Russell, S.Z. Shen, T. Nguyen, Q. Yuan, R. Varley, and S. Li, Polym. Adv. Technol., 23, 1564 (2012).

29. W.O. Yah, A. Takahara, and Y.M. Lvov, J. Am. Chem. Soc., 134, 1853 (2012).

30. M. Sahnoune, A. Taguet, B. Otazaghine, M. Kaci, and J.M. Lopez-Cuesta, Polym. Int., 66, 300 (2017).

31. T. Zheng and X. Ni, RSC Adv., 6, 57122 (2016).

32. S. Kennouche, N. Le Moigne, M. Kaci, J.C. Quantin, A. S. Caro-Bretelle, C. Delaite, and J.M. Lopez-Cuesta, Eur. Polym. J., 75, 142 (2016).

33. H. Amar, T. Braisaz, D. Villemin, and B. Moreau, Mater. Chem. Phys., 110, 1 (2008).

34. S. Mellouk, S. Cherifi, M. Sassi, K. Marouf-Khelifa, A. Bengueddach, J. Schott, and A. Khelifa, Appl. Clay Sci., 44, 230 (2009).

35. C. Breen, N. D'Mello, and J. Yarwood, J. Mater. Chem., 12, 273 (2002).

36. K. Belkassa, F. Bessaha, K. Marouf-Khelifa, I. Batonneau-Gener, J. Comparot, and A. Khelifa, Colloids Surfaces A Physicochem. Eng. Asp., 421, 26 (2013).

37. S.V. Levchik, L. Costa, and G. Camino, Polym. Degrad. Stab., 36, 31 (1992).

38. R.E. Lyon and R.N. Walters, J. Anal. Appl. Pyrolysis., 71, 27 (2004).

39. R. Sonnier, L. Ferry, C. Longuet, F. Laoutid, B. Friederich, A. Laachachi, and J.M. Lopez-Cuesta, Polym. Adv. Technol., 22, 1091 (2011).

40. A. Fina and G. Camino, Polym. Adv. Technol., 22, 1147 (2011).

41. B. Schartel and T.R. Hull, Fire Mater., 31, 327 (2007).

42. E. Joussein, S. Petit, J. Churchman, B. Theng, D. Righi, and B. Delvaux, Clay Miner, 40, 383 (2005).

43. F. Levchik, G. Camino, L. Costa, S.V. Levchik, G.F. Levchik, and A.I. Lesnikovich, Fire Mater., 20, 183 (1996).

44. B. Lecouvet, M. Sclavons, C. Bailly, and S. Bourbigot, Polym. Degrad. Stab., 98, 2268 (2013).

45. L. Dumazert, D. Rasselet, B. Pang, B. Gallard, S. Kennouche, and J.M. Lopez-Cuesta, Polym. Adv. Technol., 29, 69 (2018). 